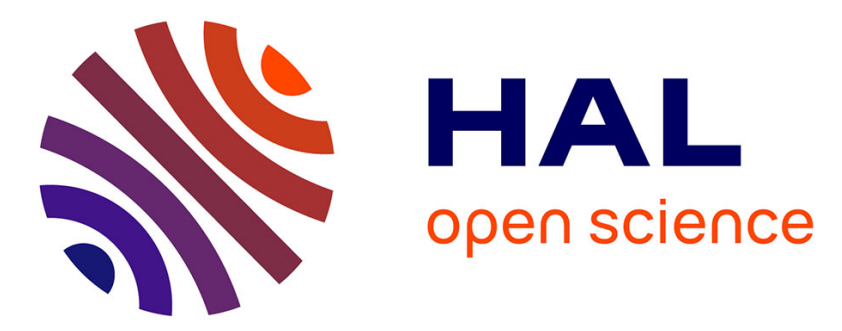

\title{
A 43pJ per Inference CBNN-based Compute-in-sensor Associative Memory in 28nm FDSOI
}

Benoit Larras, Antoine Frappé

\section{To cite this version:}

Benoit Larras, Antoine Frappé. A 43pJ per Inference CBNN-based Compute-in-sensor Associative Memory in 28nm FDSOI. ESSCIRC 2021 - IEEE 47th European Solid State Circuits Conference (ESSCIRC), Sep 2021, Grenoble, France. pp.111-114, 10.1109/ESSCIRC53450.2021.9567808 . hal03482304

\section{HAL Id: hal-03482304 https://hal.science/hal-03482304}

Submitted on 17 Dec 2021

HAL is a multi-disciplinary open access archive for the deposit and dissemination of scientific research documents, whether they are published or not. The documents may come from teaching and research institutions in France or abroad, or from public or private research centers.
L'archive ouverte pluridisciplinaire HAL, est destinée au dépôt et à la diffusion de documents scientifiques de niveau recherche, publiés ou non, émanant des établissements d'enseignement et de recherche français ou étrangers, des laboratoires publics ou privés. 


\title{
A 43pJ per Inference CBNN-based Compute- in-sensor Associative Memory in 28nm FDSOI
}

\author{
Benoit Larras and Antoine Frappé \\ Univ. Lille, CNRS, Centrale Lille, Junia, Univ. Polytechnique Hauts-de-France, UMR 8520 - IEMN, F-59000 Lille, France \\ benoit.larras@junia.com
}

\begin{abstract}
Distributed smart sensors are more and more used in applications such as biomedical or domestic monitoring. However, each sensor broadcasts data wirelessly to the others or to an aggregator, which leads to energy-hungry sensor nodes and an increased latency at the network level. To tackle both challenges, this work proposes to distribute part of the processing elements in each sensor node and presents an ASIC implementation of an associative memory using clique-based neural networks (CBNNs) coupled with an integrated SRAM memory, in a 28nm FDSOI technology node. It consumes $43 \mathrm{pJ}$ for a single inference, which is 6.5 times better than state-of-the-art associative memories implementations, for the same memory size.
\end{abstract}

\section{INTRODUCTION}

In the context of healthcare monitoring, wireless body area sensor networks (WBAN) are used to gather data in multiple places of the body. The targeted applications include cardiac monitoring, posture or gesture recognition, etc. Traditionally, a WBAN is structured as in [1], Figure 1.a. All the sensors in the network acquire data through their sensing interface and broadcast it wirelessly. Then, an aggregator gathers information from all the sensors and processes the whole data flow with one or more processing elements (PEs), usually using Artificial Intelligence (AI) algorithms. They are connected to a dedicated memory designed to store all the sensors' data, connections map, and system states.

However, several drawbacks of this architecture need to be highlighted for the case of autonomous sensors in a WBAN. First of all, the sensors transmit continuously the acquired raw data, which consumes most of the energy budget of the sensor. Besides, PEs in the aggregator have to compute the concatenated data flow, either by the means of multiple PEs operating in parallel or by increasing the computation time. The latency of the network is also impacted since the PEs have to wait to receive all the data to begin their computation. Finally, a larger bus is needed to communicate with the global memory in the aggregator, which requires increased time and energy budgets.

In the context of sensor networks, the "Near-sensor computing" paradigm tends to move at least a part of the PEs within the sensors, instead of computing multiple data flows in the same block, as described in [2], Figure 1.b. Even though the processing capability becomes more limited in each sensor if the PEs are divided among the network, there is an opportunity to decrease the sensors' energy consumption. At the sensor level, pre-processing data allows decreasing the number of bits to transmit to the rest of the network, which also decreases the sensor's total energy consumption. The memory exchanges and global latency are also reduced at the network level, which increases the capabilities of real-time operation.

In WBANs, a commonly used computation is the convergence to the closest a priori stored configuration of physiological parameters. It is implemented, in [3] in the case of hand gesture recognition, and in [4] in the case of posture recognition, by the means of associative memories. This type of AI paradigm is interesting as it does not require off-line training of the entire network, but storing the different configurations to output. In [3], the minimal Hamming distance with the stored gestures is computed with a global digital logic block from surface electromyography sensors on the arm. The memory and PE are implemented in an aggregator and are not easily distributed next to the sensors. In [4], a

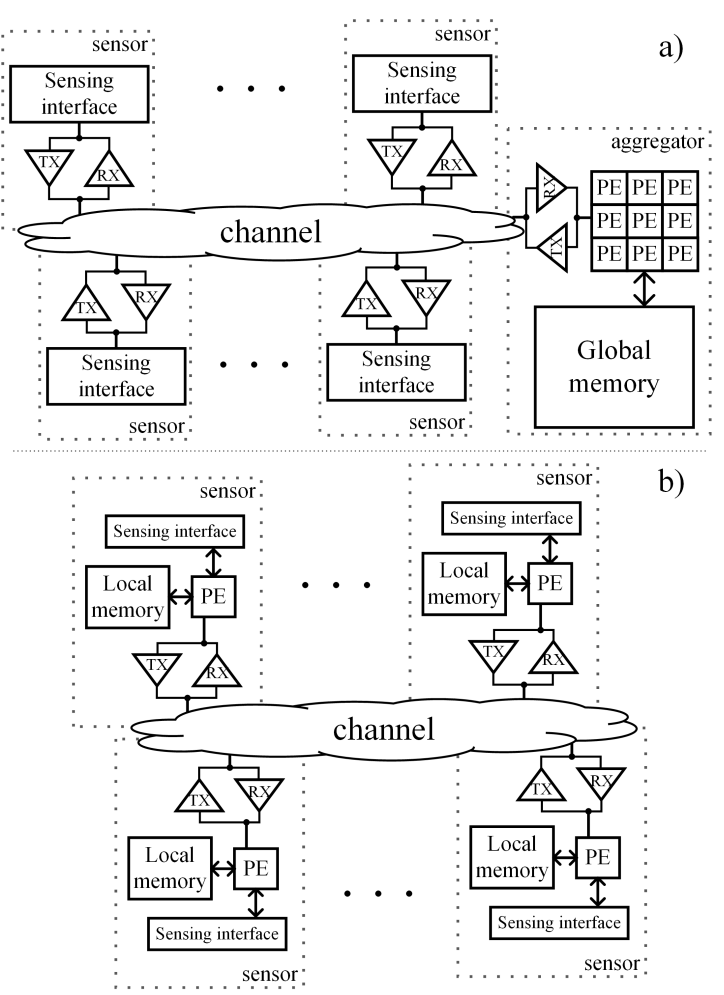

Figure 1. Structural description of 2 versions of a WBAN. a) Architecture with centralized computations in an aggregator. b) Architecture with distribution of the processing elements in the sensors. 
mixed-signal implementation of a clique-based neural network $(\mathrm{CBNN})$, distributed in clusters, is used to determine the posture by combining data from accelerometers on the body. This type of associative memory is strongly compatible with near-sensor distributed computing but the referenced circuit does not implement on-chip storage for sensor and external data. Other non-CMOS implementations of associative memories are possible using opto-electronic [5] and memristive [6] components.

This work proposes the implementation of a distributed PE integrating a cluster from a CBNN and its on-chip memory in 28nm FDSOI technology node. The benefits are:

- a reduced energy consumption per inference (less than $43 \mathrm{pJ}$ under $0.7 \mathrm{~V}$ supply) and silicon occupation of the block $\{\mathrm{PE}+$ memory $\}\left(27,600 \mu \mathrm{m}^{2}\right)$;

- a decreased circuit complexity by the use of transistor body biasing;

- a standalone operation by the means of memory integration and organization.

This paper is organized into the following sections. Section II describes the architecture of the proposed localized PE, Section III presents the measurement results, from individual characterization as well as applicative use-cases, and Section IV concludes the paper.

\section{PRocessing ElEMENT (PE) IMPLEMENTATION}

\section{A. CBNN behavior and distribution}

Clustered CBNN networks are formed by grouping neurons per category of information, Fig. 2. There are $N_{C}$ clusters, $N_{N}$ neurons per cluster and $N_{S}$ synapses per neuron. One cluster corresponds to the information contained by one sensor. One neuron corresponds to a set of parameter values from this sensor. Neurons from different clusters are connected to form a clique, and all the cliques represent the convergence options of the network. An in-cluster "Winner-Takes-All" (WTA) rule activates or not the neurons. Thus, only one neuron is activated per cluster.

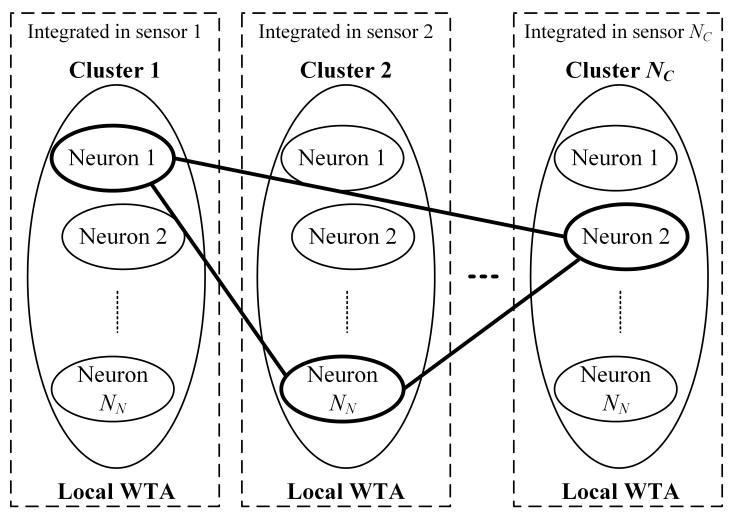

Figure 2. Structure of a CBNN implementing $N_{C}$ clusters of $N_{N}$ neurons each. Each cluster is integrated in an independent sensor. An example of a clique is highlighted in black.
The clustered structure of the CBNN is compatible with the "Near-sensor computing" paradigm, as the computations are localized per cluster. The clusters can therefore be dispatched next to the sensors as the PEs in Fig. 1.b. The transmitted data from each sensor become the output of the integrated cluster, plus its index (reference address in the network), instead of raw data from the sensors. The total number of bits transmitted by a sensor node is $\log _{2}\left(N_{C}\right)+\log _{2}\left(N_{N}\right)$. In terms of power consumption and latency, this transmission scheme is more efficient than computing all the sensor features with the whole CBNN in the aggregator, for a value of $N_{N}$ greater than 27 [4]. Moreover, the embedded memory depicted in Fig. 1 can be distributed as well in each sensor node. Each local memory can therefore be reduced to store only the incoming connections to the concerned integrated cluster. With smaller memories, the access time for each cluster is faster and thus more energy efficient.

\section{B. PE mixed-signal circuit}

Analog CMOS circuits implement a cluster of neurons with the lowest circuit complexity and, thus, lead to low power consumption [7]. $N_{S}$ synapses feed the input contributions into each neuron, then the WTA operation is triggered between the neurons in the cluster, as shown in Fig. 3. The synapses are implemented as switched current sources converting binary input voltages into binary currents, i.e. $0 \mathrm{~A}$ or $I_{\mathrm{UNIT}}$, which are summed at node $\mathrm{A}$.

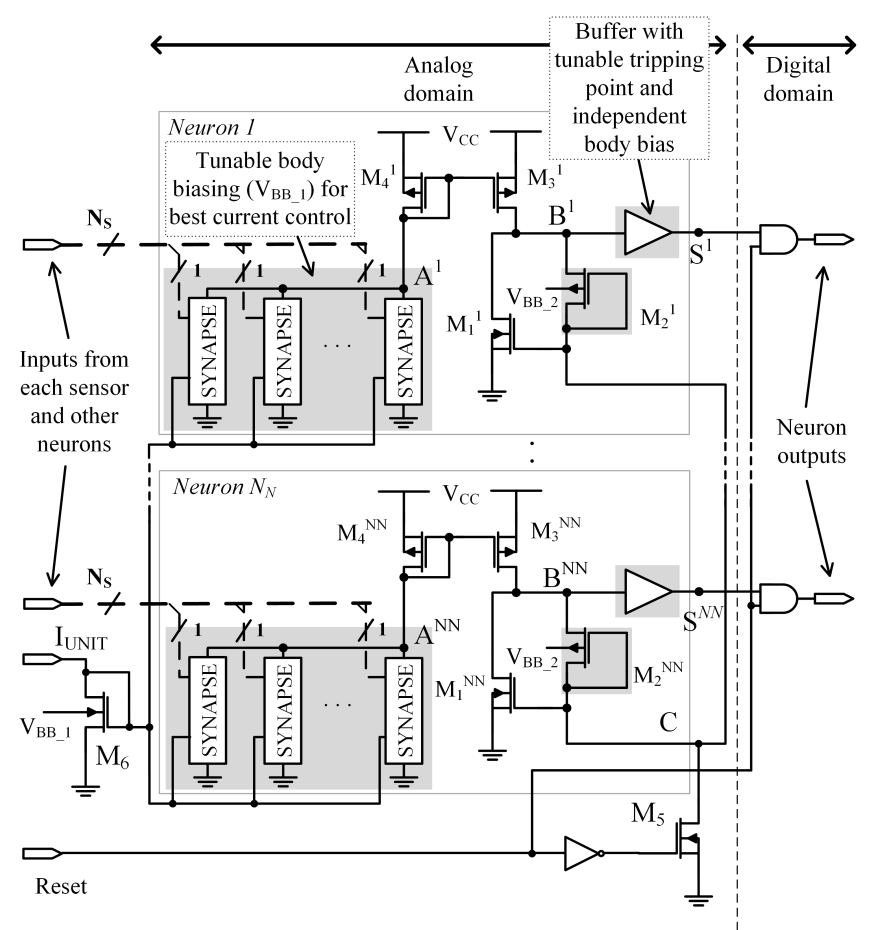

Figure 3. Schematic of a mixed-signal cluster of $N_{N}$ neurons in a CBNN. $N_{S}$ is the number of possible input connection per neuron. Shaded areas show independently body-biased transistors. 
The WTA circuit, separated in the neurons, is composed of transistors $\mathrm{M}_{1}$ and diodes $\mathrm{M}_{2}$, and receives the input current copied by the $\mathrm{M}_{3}-\mathrm{M}_{4}$ current mirror. The current flowing in the WTA element sets the drain-source voltage of $\mathrm{M}_{1}$ depending on the currents flowing in the other neurons, connected by the means of node $\mathrm{C}$. The result of the operation is output as a binary voltage at node $\mathrm{B}$. If the input current of a neuron is the highest, the drain-source voltage of the corresponding transistor $\mathrm{M}_{1}$ is set higher than its saturation voltage. Otherwise, the drain-source voltage of $\mathrm{M}_{1}$ is set under its saturation voltage. A buffer translates the voltage at node $\mathrm{B}$ in a standard binary voltage at node $\mathrm{S}$. The activated neuron's index is output to the rest of the network.

The circuit, designed for the 28nm FDSOI technology node, benefits from the body biasing feature in 3 ways. First, in order to optimize the energy consumption of the PE, the transistors in the synapses operate in the subthreshold region. A better control of the $I_{\mathrm{UNIT}}$ current value is achieved through body biasing of the transistors in the synapses. Second, the buffer threshold at node B is affected by PVT variations and typically requires a feedback circuit to calibrate the threshold by current starving. Here, the buffer is body-biased independently so that the threshold is always comprised between the high and low levels of the WTA circuit, and thus no external circuitry is needed. Finally, leakage currents flowing in each blocked diode $\mathrm{M}_{2}$ are drawn from the synapses currents and limit the number of WTA elements in parallel. A second stage of WTA operation is needed for higher number of neurons in a cluster [7]. By body biasing separately the diodes, the leakage current is reduced from $3.6 \mathrm{nA}$ down to $41 \mathrm{pA}$, which can allow

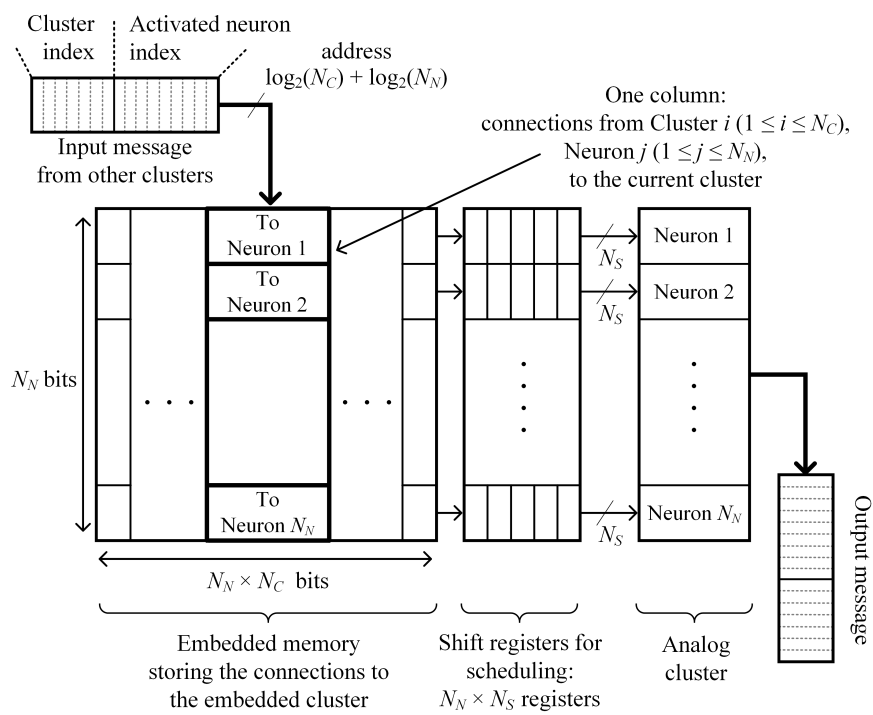

Figure 4. Organization of the embedded memory. It contains $N_{C} \times N_{N}$ words of $N_{N}$ bits each, displayed as columns. Each square represents a bit storing the connection state between 2 neurons from a distant cluster to the concerned integrated cluster. Each row represents a neuron in the concerned cluster (destination neuron), and each column a neuron in the whole network (source neuron) either further reducing $I_{\mathrm{UNIT}}$, or increasing the number of WTA elements in parallel by a factor of 100 .

\section{Memory organization}

The embedded memory stores the binary connections between the neurons from different clusters in the network. The only implemented connections are those from neurons of other clusters to the considered integrated cluster, as shown in Fig. 4. The memory stores $N_{C} \times N_{N}$ words. It corresponds to the number of neurons in the whole network. Each word contains $N_{N}$ bits, each bit stimulating one neuron in the considered cluster.

The reading process is scheduled as follows. The circuit receives $\log _{2}\left(N_{C}\right)+\log _{2}\left(N_{N}\right)$ bits from another cluster. It corresponds to the index winning neuron in that cluster, concatenated with the cluster index. This message is used as an address to output the activated connections from the embedded memory to the current cluster. The output word is pushed in a shift register in order to gather the following messages from the other clusters. The selected bits are input in the analog cluster at the same time, as soon as all the clusters inputs have been received. Therefore, the order in which the messages are received does not matter. A cluster can also time out before sending data, a binary timeout flag is triggered. In that case, the shift register outputs the received data as-is into the analog cluster. If half of the neurons are stimulated, a stored clique can be recovered thanks to the high inherent redundancy of CBNNs.

\section{MEASUREMENT RESULTS}

The proposed design is implemented on a $1-\mathrm{mm}^{2}$ ASIC in the $28 \mathrm{~nm}$ FDSOI technology node, as depicted in Fig. 5. Three independent instances of the block $\{\mathrm{PE}+$ memory $\}$, named $\mathrm{C} 1$, C2 and C3, are implemented on-chip. They can be used for different sensors in the same network.

\section{A. Characterization of the block $\{P E+$ memory $\}$}

Each block implements a cluster of 32 neurons, with 16 synapses per neuron. There are 512 scheduling registers per block, and the memory stores 512 words of 32 bits each. The total silicon area per block $\{\mathrm{PE}+$ memory $\}$ is $27,600 \mu \mathrm{m}^{2}$.
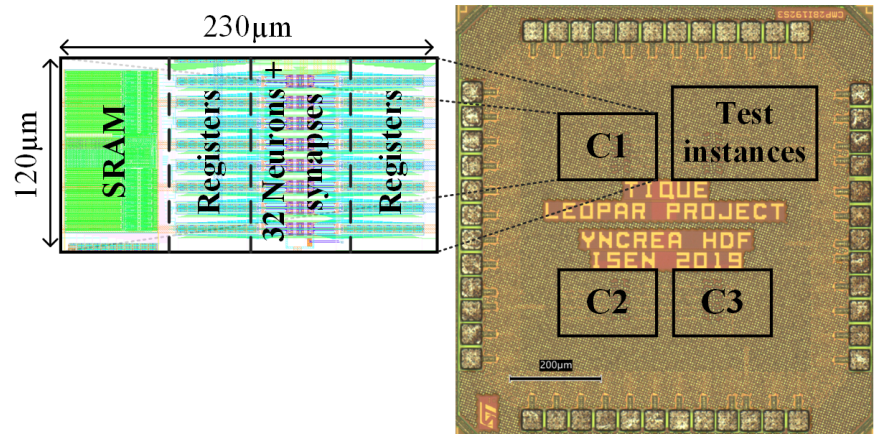

Figure 5. Photograph of the fabricated chip in 28nm FDSOI technology node. It implements 3 independent blocks \{PE+memory $\} \mathrm{C} 1, \mathrm{C} 2$ and $\mathrm{C} 3$. 
Table I

ELECTRICAL CHARACTERISTICS OF A BLOCK \{PE+MEMORY

\begin{tabular}{|c|c|c|}
\hline Technology process & \multirow{2}{*}{\multicolumn{2}{|c|}{$\frac{28 \mathrm{~nm} \text { FDSOI }}{30 \mathrm{nA}}$}} \\
\hline Unitary synapse current $I_{\mathrm{UNIT}}$ & & \\
\hline Supply voltage $V_{\mathrm{CC}}$ & $1 \mathrm{~V}$ & $0.7 \mathrm{~V}$ \\
\hline Static power consumption & $7 \mu \mathrm{W}$ & $0.8 \mu \mathrm{W}$ \\
\hline Energy per memory read & $3.6 \mathrm{pJ}$ & $2.5 \mathrm{pJ}$ \\
\hline Energy consumption: registers & $\max .19 \mathrm{pJ}$ & $\max .2 .4 \mathrm{pJ}$ \\
\hline Energy consumption: analog cluster & $\max .820 \mathrm{fJ}^{*}$ & $\max .550 \mathrm{fJ}^{*}$ \\
\hline Analog cluster inference time & \multicolumn{2}{|c|}{$101 \mathrm{~ns}$} \\
\hline
\end{tabular}

The electrical characteristics are shown in Table I. The circuit operates at a supply voltage $V_{\mathrm{CC}}$ between $0.7 \mathrm{~V}$ and $1 \mathrm{~V}$, with a unitary current $I_{\mathrm{UNIT}}$ of $30 \mathrm{nA}$. The measured static power consumption for one instance is of $7 \mu \mathrm{W}$ for $1 \mathrm{~V}$, and $0.8 \mu \mathrm{W}$ for $0.7 \mathrm{~V}$. Once the memory is loaded, a series of up to 16 32-bit vectors is loaded in the shift registers, and the data bits are loaded in the cluster, starting its convergence phase. The power consumption of the analog WTA depends on the number of ' 1 ' in the memory, however most of the dynamic power is used in the shift registers. Including the memory read operations, the block $\{\mathrm{PE}+$ memory $\}$ consumes at most $43 \mathrm{pJ}$ per inference under $0.7 \mathrm{~V}$ supply.

\section{B. State of the art of associative memories for WBAN}

The individual characteristics of the proposed implementation are compared to state-of-the-art associative memories for WBAN. Their features are summed up in Table II. In [3], the associative memory used for hand gesture recognition computes the Hamming distance between the sensors' inputs and the stored configurations. However, the associative memory is implemented on an external FPGA, which constrains the system for embedded operation and is not relevant for performance comparison. A state-of-the-art associative memory implementing a multi-context ternary content addressable memory (T-CAM) is integrated in an ASIC used for speech recognition in [8]. It is combined with an SRAM storing $96 \mathrm{~kb}$ of data. The association operation consumes $80 \mathrm{pJ}$, but the main drawbacks of this implementation are linked to the use of a global memory storing all the convergence options. The static energy consumption of the global memory is 20 times more important than that of the associative operation itself, and its silicon area increases dramatically the circuit surface. Distributing the memory mitigates these drawbacks, which is not demonstrated in [4] because the memory is implemented externally on a FPGA. Globally, for a distribution in 16 sensor nodes, the energy consumption normalized to the embedded memory size is reduced in this work by a factor of 6.5. In addition, it has been shown in [4] that the energy consumption overhead due to the wireless interface is mitigated since a lower number of bits is transmitted in the distributed scheme.

\section{CONCLUSION}

This paper presents a distributed mixed-signal implementation of clique-based neural networks, combined with an SRAM memory. The circuit is used as an associative memory in
Table II

ASSOCIATIVE MEMORIES STATE OF THE ART

\begin{tabular}{|c|c|c|c|c|}
\hline & [3] & [8] & [4] & This work \\
\hline Application & $\begin{array}{c}\text { Hand } \\
\text { gesture } \\
\text { recognition }\end{array}$ & $\begin{array}{l}\text { Speech } \\
\text { recognition }\end{array}$ & $\begin{array}{l}\text { Posture } \\
\text { recognition }\end{array}$ & WBAN \\
\hline $\begin{array}{l}\text { Integration } \\
\text { process }\end{array}$ & FPGA & $\begin{array}{c}\text { ASIC } \\
65 \mathrm{~nm} \text { bulk }\end{array}$ & $\begin{array}{c}\text { ASIC } \\
65 \mathrm{~nm} \text { bulk } \\
+ \text { FPGA }\end{array}$ & $\begin{array}{c}\text { ASIC } \\
\text { 28nm } \\
\text { FDSOI } \\
\end{array}$ \\
\hline $\begin{array}{l}\text { Computation } \\
\text { type }\end{array}$ & $\begin{array}{l}\text { Hamming } \\
\text { distance } \\
\text { digital } \\
\text { computa- } \\
\text { tion }\end{array}$ & $\begin{array}{l}\text { Multi- } \\
\text { context } \\
\text { T-CAM }\end{array}$ & CBNN & CBNN \\
\hline Topology & Localized & Localized & Distributed & Distributed \\
\hline $\begin{array}{l}\text { Available } \\
\text { memory }\end{array}$ & - & $96 \mathrm{~kb}$ & $\begin{array}{l}8 \mathrm{~kb}(40 \mathrm{~kb} \\
\text { for } 5 \\
\text { dist.nodes) }\end{array}$ & $\begin{array}{c}\mathbf{1 6 k b} \\
\text { (256kb for } \\
16 \text { dist. } \\
\text { nodes) }\end{array}$ \\
\hline Silicon area & n.a. & $\begin{array}{c}174,000 \mu \mathrm{m}^{2} \\
\text { without } \\
\text { SRAM }\end{array}$ & $\begin{array}{c}210,000 \mu \mathrm{m}^{2} \\
\text { without } \\
\text { SRAM }\end{array}$ & $27,600 \mu \mathbf{m}^{2}$ \\
\hline $\begin{array}{l}\text { Core energy } \\
\text { consumption }\end{array}$ & n.a. & $80 \mathrm{pJ}$ & $\max .18 \mathrm{pJ}$ & $\max .3 p J^{*}$ \\
\hline $\begin{array}{l}\text { Total energy } \\
\text { consumption }\end{array}$ & - & $1.68 \mathrm{~nJ}$ & - & $\begin{array}{l}\text { max. } 43 p J \\
\text { per node* }\end{array}$ \\
\hline $\begin{array}{c}\text { Energy } \\
\text { consumption } \\
\text { per stored } \\
\text { bit }\end{array}$ & - & $17.5 \mathrm{pJ} / \mathrm{bit}$ & - & $\begin{array}{c}\max . \\
2.7 \mathrm{pJ} / \mathrm{bit}\end{array}$ \\
\hline
\end{tabular}

* obtained for $0.7 \mathrm{~V}$.

WBAN applications, and has been fabricated in 28nm FDSOI technology node. It consumes at most $43 \mathrm{pJ}$ per inference, which is 6.5 times the energy consumption of state-of-theart implementation of associative memories such as T-CAMs for the same memory size. Further work will include real-life demonstration of the complete WBAN with distributed PEs and memories in the sensors.

\section{REFERENCES}

[1] S. Movassaghi et al., "Wireless Body Area Networks: A Survey," IEEE Communications Surveys Tutorials, vol. 16, no. 3, pp. 1658-1686, 2014.

[2] W. Yu et al., "A survey on the edge computing for the internet of things," IEEE Access, vol. 6, pp. 6900-6919, 2018.

[3] A. Moin et al., "A wearable biosensing system with in-sensor adaptive machine learning for hand gesture recognition," Nature Electronics, vol. 4, pp. 54-63, 2021.

[4] B. Larras and A. Frappé, "On the Distribution of Clique-Based Neural Networks for Edge AI," IEEE Journal on Emerging and Selected Topics in Circuits and Systems, pp. 1-1, 2020.

[5] Z. Wang and X. Wang, "A novel memristor-based circuit implementation of full-function pavlov associative memory accorded with biological feature," IEEE Transactions on Circuits and Systems I: Regular Papers, vol. 65, no. 7, pp. 2210-2220, 2018.

[6] Y. Alkabani, M. Miscuglio, V. J. Sorger, and T. El-Ghazawi, "Oe-cam: A hybrid opto-electronic content addressable memory," IEEE Photonics Journal, vol. 12, no. 2, pp. 1-14, 2020.

[7] B. Larras et al., "A Fully Flexible Circuit Implementation of Clique-Based Neural Networks in 65-nm CMOS," IEEE Transactions on Circuits and Systems I: Regular Papers, vol. 66, no. 5, pp. 1704-1715, May 2019.

[8] R. Arakawa et al., "Multi-Context TCAM-Based Selective Computing: Design Space Exploration for a Low-Power NN," IEEE Transactions on Circuits and Systems I: Regular Papers, vol. 68, no. 1, pp. 67-76, 2021. 